\title{
Transanal endorectal pull-through for classic segment Hirschsprung's disease: With or without laparoscopic mobilization of the rectosigmoid?
}

\author{
Teun J. van de Ven ${ }^{a, b, *}$, Cornelius E.J. Sloots ${ }^{a}$, Marc H.W.A. Wijnen ${ }^{b}$, \\ Roxana Rassouli $^{b}$, Iris van Rooij ${ }^{c}$, Rene M. Wijnen ${ }^{a}$, Ivo de Blaauw ${ }^{a, b}$ \\ a Department of Pediatric Surgery, Erasmus MC - Sophia Children's Hospital, Rotterdam, The Netherlands \\ ${ }^{\mathrm{b}}$ Department of Surgery - Pediatric Surgery, Radboud University Nijmegen Medical Center, Nijmegen, The Netherlands \\ ${ }^{\mathrm{c}}$ Department of Epidemiology, biostatistics and HTA, Radboud University Nijmegen Medical Centre, Nijmegen, The Netherlands
}

Received 21 August 2012; revised 11 April 2013; accepted 13 April 2013

\author{
Key words: \\ Hirschsprung's disease; \\ TERPT; \\ TEPT; \\ Surgery; \\ Laparoscopic; \\ Postoperative outcomes
}

\begin{abstract}
Background: It has been suggested that the outcome of transanal endorectal pull-through for classic Hirschprung's disease can be improved by laparoscopically mobilizing the colon before the pullthrough. Methods: Charts of 43 patients (2005-2009) with proven recto-sigmoid aganglionosis were retrospectively analyzed with respect to postoperative outcomes. Twenty-one had been treated with the transanal endorectal pull through (TERPT) and 22 with the laparoscopically assisted TERPT (LTERPT). Results: Gender ratio, congenital anomalies, preoperative enterostomy, and follow up did not differ between the groups. More colon was resected in the TERPT group: median $25 \mathrm{~cm}$ vs. $15 \mathrm{~cm}$ in the LTERPT group $(\mathrm{p}<0.001)$. The TERPT-procedure took less time: median $153 \mathrm{~min}$. vs. L-TERPT $263 \mathrm{~min}(\mathrm{p}<0.001)$. Postoperatively, three patients showed colonic torsions after TERPT $(\mathrm{p}=0.07)$. The long-term clinical outcomes did not differ significantly between both groups. There was a significant association between length of resection and obstructive symptoms $(\mathrm{OR}=0.92, \mathrm{p}=0.01)$.

Conclusion: Postoperative and clinical outcomes are similar using the TERPT or L-TERPT to correct classic segment Hirschsprung's disease. Prevention of colonic torsion should be the prime concern during the TERPT procedure. L-TERPT requires laparoscopic equipment and takes more operation time, whereas TERPT leaves no visible scars. The positive relation between the larger length of resection and obstructive symptoms requires additional research.

(C) 2013 Elsevier Inc. All rights reserved.
\end{abstract}

There has been a major development in the surgical treatment of classic segment Hirschsprung's disease after the introduction of the one-stage transanal endorectal pull-through.

* Corresponding author. Department of Surgery - Pediatric Surgery, PB 9101, 6500 HB, Nijmegen, The Netherlands. Tel.: +31 243619761; fax: +31243613547.

E-mail address: T.vandeven@kchir.umcn.nl (T.J. van de Ven).
Previous to this procedure, the most commonly used pullthrough techniques were the Swenson, Soave or Duhamel pullthrough [1]. In the 90s two major new approaches were developed: the laparoscopic approach in which the transition zone can be identified, biopsies taken and the rectum and sigmoid mobilized before pull-through [2-4]. The other technique is the complete transanal endorectal pull-through introduced by de La Torre and Langer $[5,6]$. Both techniques 
have proven to be preferable to the open techniques [7-10]. It has not been clarified whether the best results are accomplished by the laparoscopically assisted transanal pull-through or the complete transanal pull-through. In two tertiary referral centers two different pull-through procedures are performed. At Radboud University Nijmegen Medical Centre (RUNMC) a complete transanal endorectal pullthrough (TERPT) is performed, whereas at Erasmus Medical Centre Sophia Children's Hospital (EMC-Sophia) colon biopsy and mobilization of the colon are performed laparoscopically before the final pull-through (L-TERPT).

This study aims to investigate the best surgical treatment for classic segment Hirschsprung's disease (concerning the early- and late post-operative outcomes) by comparing these two procedures. Should the transanal endorectal pull-through for classic segment Hirschsprung's disease be performed with or without laparoscopic mobilization of the colon before the pull-through?

\section{Methods}

\subsection{Study population}

All patients with classic segment Hirschsprung's disease who were treated at the EMC-Sophia or the RUNMC with LTERPT or TERPT between 2005 and 2009. Patients with a follow-up of more than 3 months and proven aganglionosis of the rectosigmoid were included. Forty-three patients were eligible: 22 treated with L-TERPT and 21 with TERPT.

\subsection{Surgical techniques}

The TERPT is a transanal endorectal pull-through as described by de la Torre and Langer [5,6]. Full thickness biopsies are taken and analyzed during the pull-through procedure. The L-TERPT is a laparoscopically assisted TERPT procedure as described by Georgeson [2]. Laparoscopy is used to take full thickness biopsies and to mobilize the colon. As described by de la Torre, a rectal cuff (muscle sleeve) is dissected from the submucosa and left behind. The ganglionic bowel is then pulled through this rectal cuff [1]. Recently, it has been suggested to decrease the length of the rectal cuff (in both techniques) to a length of 1-2 centimeters. Currently this is the standard of care by most pediatric surgeons. There is a team of 3 surgeons at the RUNMC (TERPT) and 5 surgeons at the EMC-Sophia (L-TERPT). The biopsies are frozen and analyzed, during surgery, by an experienced pathologist. For pathology analysis we use calretinin staining to decrease potential inter-observer bias.

\subsection{Follow-up}

Patients were seen postoperatively by the pediatric surgeon. The frequency of visits depended on the amount of care they needed. Three weeks postoperatively all surgeons examined the patients. If an anal stenosis was diagnosed, dilatations with Hegar dilators were started on daily basis. In both centers, prevention of constipation and obstructive symptoms was actively managed with laxatives and bowel management. The main goal of bowel management is the prevention of obstructive symptoms and enterocolitis. Bowel management will, at the age of potty training, be used to treat incontinence. Depending on the consistency of the stool and the severity of the constipation, laxatives or enemas are prescribed or bowel irrigations are started using a Foley catheter and saline to achieve at least one bowel movement per day.

\subsection{Data collection}

The charts of the patients were reviewed for congenital anomalies, age at surgery, preoperative ileostomy or colostomy and length of follow up. Further, surgical characteristics were reviewed for length of resection, operative time and length of hospital stay. Early postoperative outcomes, meaning less than 30 days, were analyzed by scoring: colonic torsion, redo laparotomy, wound infection, pneumonia, sepsis, stenosis, leakage, ileus and enterocolitis. Long-term postoperative outcomes (after 30 days) were analyzed by scoring: obstructive symptoms, need for dilatation, need for enemas, need for laxatives, and mortality. Obstructive symptoms were defined as: a period of enterocolitis (using the Delphi score [11]), need for dilatation, stenosis and constipation. Constipation was defined as: need for laxatives, enemas and/or bowel irrigations longer than 3 months.

\subsection{Statistical analysis}

Differences between both surgical procedures were analyzed. The Fischer exact test was used to analyze the dichotomous variables and the Mann-Whitney $U$ test was used to analyze the continuous parameters. The influence of the length of resection on obstructive symptoms was investigated using a multivariate logistic regression analysis. Potential confounders, such as type of surgery, redo surgery, and age at surgery, were adjusted in a multivariate logistic regression analysis.

\section{Results}

Table 1 shows the patients and surgical characteristics. There were no differences in gender, congenital anomalies and pre-operative ileostomy or colostomies. The median age at surgery was 4 months in the L-TERPT group versus 2 months in the TERPT group $(p=0,05)$. The median follow up time after L-TERPT and TERPT was comparable (48 vs. 46 months, respectively). Surgical characteristics 
Table 1 General patient and surgical characteristics.

\begin{tabular}{llll}
\hline & L-TERPT $\mathrm{n}=22$ & TERPT n $=21$ & $\mathrm{p}-\mathrm{Value}$ \\
\hline Male:female & $17: 5$ & $17: 4$ & 0.77 \\
Down syndrome and Waardenburg syndrome, $\mathrm{n}(\%)$ & $3(14)$ & $2(10)$ & $2(10)$ \\
Cardiac congenital anomalies, n (\%) & $0(0)$ & $1(5)$ & 0.67 \\
Other congenital anomalies, n (\%) & $1(5)$ & $3(14)$ & 0.14 \\
Ileo-/colostomy, n (\%) & $4(18)$ & $2.4(0.7-31.6)$ & $5.4(3.3-12.5)$ \\
Median age at surgery in months (range) & $4.0(1.5-43.8)$ & $46(24-76)$ & 0.97 \\
Median weight at surgery in kg (range) & $6.5(4.7-14.5)$ & $153(103-311)$ & 0.05 \\
Median follow-up time after surgery in months (range) & $48(10-71)$ & $25(14-50)$ & 0.03 \\
Median surgery time in minutes (range) & $263(175-410)$ & $6(3-23)$ & 0.66 \\
Median length of resection in cm (range) & $15(6-40)$ & $6(3-53)$ & $<0.001$ \\
Median length of hospital stay in days (range) & & 0.29 \\
\hline ns, not statistically significant. & &
\end{tabular}

showed a significant difference for length of resection. The median length of resection after L-TERPT was much shorter compared to TERPT ( 15 vs. $25 \mathrm{~cm}$, respectively, p < 0,001). However, surgical time was much longer with L-TERPT compared to TERPT (median of 263 minutes vs. 153 minutes, respectively, $\mathrm{p}<0,001$.). The median length of hospital stay was similar in both groups, both 6 days. Early postoperative complications showed no significant differences (Table 2). However, there seemed to be more colonic torsions after TERPT. All 3 redo laparotomies after TERPT were performed one month post operatively and all indicated by the mentioned colonic torsions. Long term postoperative outcomes and complications showed no significant differences between both groups (Table 3). Three patients had enterocolitis after L-TERPT versus 5 after TERPT and dilatation was more often indicated after TERPT, although these differences are not statistically different. Logistic regression analyses showed a significant association between length of resection and obstructive symptoms $(\mathrm{p}=0.01$, $\mathrm{OR}=0.92,95 \%$ CI (0.86-0.99)), even after adjustment for type of surgery, colonic torsion, or age at surgery. The chance of obstructive symptoms is $8 \%$ higher in patients with a small length of resection. In both groups 10 patients did not need laxatives, enemas or bowel management.

Table 2 Early postoperative complications ( $<30$ days after surgery).

\begin{tabular}{llll}
\hline & $\begin{array}{l}\text { L-TERPT } \\
\mathrm{n}=22\end{array}$ & $\begin{array}{l}\text { TERPT } \\
\mathrm{n}=21\end{array}$ & p-value \\
\hline Wound infection, n (\%) & $1(5)$ & $0(0)$ & 0.32 \\
Leakages, n (\%) & $1(5)$ & $0(0)$ & 0.32 \\
Stenosis, n (\%) & $1(5)$ & $0(0)$ & 0.32 \\
Ileus, n (\%) & $0(0)$ & $1(5)$ & 0.30 \\
Pneumonia, n (\%) & $0(0)$ & $0(0)$ & $\mathrm{ns}$ \\
Sepsis, n (\%) & $0(0)$ & $0(0)$ & $\mathrm{ns}$ \\
Enterocolitis, n (\%) & $0(0)$ & $1(5)$ & 0.30 \\
Torsion of colon, n (\%) & $0(0)$ & $3(14)$ & 0.07 \\
Mortality, n (\%) & $0(0)$ & $0(0)$ & $\mathrm{ns}$ \\
\hline ns, not statistically significant. & &
\end{tabular}

\section{Discussion}

The transanal endorectal pull-through technique and laparoscopically assisted TERPT seem to be comparable concerning most early postoperative and long term outcomes such as leakage, stenosis, infection and obstructive symptoms. However there was a remarkable difference in length of resection, being smaller after the L-TERPT. A similar difference in length of resection was seen when the open Rehbein procedure was compared to the transanal approach [7]. In this study it was suggested that the macroscopic view of the transition zone during laparotomy appears to result in less resection of bowel. In the transanal pull-through technique the visibility of the transition zone may be less, leading to more resection to ensure having resected all aganglionic colon. The surgeons who used the TERPT prefer to resect at least 10 centimeters proximal to the positive ganglionic biopsy. Resection of such a large margin of ganglionic bowel was preferred in order to prevent leaving the transition zone behind. How much ganglionic bowel should be resected is still controversial in literature but ranges from 5 to $15 \mathrm{~cm}$ above normal ganglionic biopsy [1] Logistic regression analysis provided a small negative association between length of resection and obstructive

Table 3 Long term outcome and complications ( $>30$ days after surgery).

\begin{tabular}{lccl}
\hline & $\begin{array}{c}\text { L-TERPT } \\
\mathrm{n}=22\end{array}$ & $\begin{array}{c}\text { TERPT } \\
\mathrm{n}=21\end{array}$ & $\mathrm{p}$-value \\
\hline Enterocolitis, n (\%) & $3(14)$ & $5(24)$ & 0.39 \\
Obstructive symptoms, n (\%) & $11(50)$ & $8(38)$ & 0.43 \\
Need for laxatives, n (\%) & $9(41)$ & $8(38)$ & 0.85 \\
Need for dilatation, n (\%) & $1(5)$ & $4(19)$ & 0.14 \\
$\begin{array}{l}\text { Need for bowel management } \\
\text { or enema's, n (\%) }\end{array}$ & $11(50)$ & $9(43)$ & 0.64 \\
$\begin{array}{l}\text { No need for laxatives, enema's } \\
\quad 10(45)\end{array}$ & $10(48)$ & 0.89 \\
$\begin{array}{l}\text { Mor bowel management, n (\%) } \\
\text { Mortality, n (\%) }\end{array}$ & $0(0)$ & $0(0)$ & $\mathrm{ns}$ \\
\hline
\end{tabular}

ns, not statistically significant. 
symptoms, suggesting that we should resect a larger part of ganglionic bowel above the transition zone to avoid obstructive symptoms. However, this effect may be limited as only a slight trend of less obstructive symptoms was seen in the TERPT group. Moreover, the amount of patients without the need for laxatives was equal in both groups. Based on our experience, we still recommend dissecting at least 10 centimeters of colon above the ganglionic biopsy. The L-TERPT procedure took on average 109 minutes longer than the TERPT procedure. Clearly, it is well known that laparoscopic procedures take more time than open procedures. Another explanation of the difference in time is the time lost for frozen section biopsy results to become clear. During L-TERPT a biopsy was taken and the results were awaited before continuing the surgical procedure. With TERPT the biopsies were taken, processed and, while the results were awaited, the surgeon proceeded with the transanal endorectal procedure. This saved some time and may explain the time difference.

Post operative complications can be divided into preventable (leakage, infections, residual aganglionosis), partially preventable (residual constipation, dermatitis) and unpreventable (enterocolitis). Although not significant, we did observe more preventable complications (colonic torsions) after TERPT. Both partially preventable and unpreventable complications were similar in both groups concerning short and long term outcomes.

The 3 colonic torsions strongly suggest that this complication is more common when performing TERPT. The colonic torsions that occurred after TERPT may be influenced by the learning curve, although all procedures were not within the first cases performed by these surgeons, and the colon was marked during the dissection in order to prevent torsion. In all three cases a subtle $45-90^{\circ}$ rotation occurred resulting in obstructive symptoms but not into a complete stoppage. During re-laparoscopy and re-laparotomy the torsions were confirmed. The colo-anal anastomosis was dissected, the colon was placed in a proper position and a new anastomosis was performed under laparoscopic vision. From there, the colon was marked every $2-3 \mathrm{~cm}$, during transanal dissections to prevent torsion, as suggested by de la Torre and Langer [1]. The absence of torsions in the laparoscopic assisted pull-through was due to good intra-abdominal visibility- making it possible to check for colonic torsion just before the colo-anal anastomosis was constructed. Looking at the available literature, we found one study reporting a single colonic torsion after 25 TERPT procedures [13]. However, de la Torre and Langer do point out the risk of colonic torsion in their review on surgical techniques of the transanal approach for Hirschsprung's disease [1]. Colonic torsion is considered to be part of the learning curve of the TERPT procedure, but, as seen in our study, even beyond the learning curve there is a risk of torsion of the colon. It may still be an under-reported phenomenon.

The main limitation of this study is that the two different techniques are used in two different centers. Differences in protocols between these two centers have resulted in differences in age at surgery. The median age in the LTERPT was 4.0 months whereas it was 2.4 months in the TERPT group. However, literature shows no differences in surgical outcomes when operated at different ages for Hirschsprung's disease [12]. The median age at surgery in both groups was within the same range (and below 10 months). We therefore do not expect this to influence our parameters by age differences. Other aspects of the protocols, such as pre- and post-operative care, were similar. Another limitation of the study is the number of surgeons that operated on these patients. Although all patients are operated by two experienced surgeons we agree that this can result in significant bias. Although the advantage of comparing two centers in a retrospective study is the prevention of selection bias: patients are not referred to tertiary centers based on the technique but rather on location.

Postoperative and clinical outcomes are similar using the L-TERPT or TERPT to correct classic segment Hirschsprung's disease. The prevention of torsion of the colon after TERPT should still be the focus of attention during this procedure. The L-TERPT requires laparoscopic equipment and takes more operative time. The TERPT on the other hand leaves no visible scars. For a founded conclusion concerning the association between length of resection and obstructive symptoms, we will study the resected bowel to extricate the relation between the margin of the transition zone and obstructive symptoms.

\section{References}

[1] De La Torre L, Langer JC. Transanal endorectal pull-through for Hirschsprung disease: technique, controversies, pearls, pitfalls, and an organized approach to the management of postoperative obstructive symptoms. Semin Pediatr Surg 2010;19:96-106.

[2] Georgeson KE, Cohen RD, Hebra A, et al. Primary laparoscopicassisted endorectal colon pull through for Hirschsprung's disease: a new gold standard. Ann Surg 1999;229:678-83.

[3] Travassos DV, Bax NM, van der Zee DC. Duhamel procedure: a comparative retrospective study between an open and a laparoscopic technique. Surg Endosc 2007;21:2163-5.

[4] Durran TJ, Raffensperger JG. Laparoscopic Swenson pull-through: a comparison with the open procedure. J Pediatr Surg 1996;31: 1155-6.

[5] De La Torre-Mondragon L, Ortega-Salgado JA. Transanal endorectal pull-through for Hirschsprung's disease. J Pediatr Surg 1998;33: 1283-6.

[6] Langer JC, Minkes RK, Mazziotti MV, et al. Transanal one-stage Soave procedure for infants with Hirschsprung disease. J Pediatr Surg 1999;34:148-52.

[7] Visser R, van de Ven TJ, van Rooij IALM, et al. Is the Rehbein procedure obsolete in the treatment of Hirschsprung's disease. Pediatr Surg Int 2010;26:1117-22.

[8] Langer JC, Seifert M, Minkes RK. One-stage Soave pull-through for Hirschsprung disease: a comparison of the transanal vs open approaches. J Pediatr Surg 2000;355:820-2.

[9] De la Torre L, Ortega A. Transanal versus open endorectal pullthrough for Hirschsprung's disease. J Pediatr Surg 2000;35:1630-2. 
[10] Langer JC, Durrant AC, de la Torre L, et al. One-stage transanal Soave pullthrough for Hirschsprung disease: a multicenter experience with 141 children. Ann Surg 2003;238:569-83.

[11] Pastor AC, Osman F, Teitelbaum DH, et al. Development of a standardized definition for Hirschsprung's-associated enterocolitis: a Delphi analysis. J Pediatr Surg 2009;44:251-6.
[12] Pini Prato A, Gentilino V, Giunta C, et al. Hirschsprung disease: do risk factors of poor surgical outcome exist? J Pediatr Surg 2008;43: 612-9.

[13] Obermayr F, Szavay P, Beschorner R, et al. Outcome of transanal endorectal pullthrough in patients with Hirschsprung's disease. Eur J Pediat Surg 2009;19:220-30. 